\title{
Ejercicios de bajo impacto en prevención de complicaciones por inactividad física en niños con leucemia linfoblástica aguda
}

Low-impact exercises to prevent complications from physical inactivity in children with acute lymphoblastic leukemia

\section{Exercícios de baixo impacto para prevenir complicações de inatividade física em crianças com leucemia linfoblástica} aguda

\author{
María Fernanda Agurto Silva \\ mafer_agurto@hotmail.com \\ ORCID: 0000-0002-3319-1646 \\ Jenny Maricela Chávez Chacón \\ jennychavez211@hotmail.com \\ ORCID: 0000-0003-1798-2250
}

\author{
María De Los Ángeles Bobadilla Cárdenas \\ angelesbc97@gmail.com \\ ORCID: 0000-0001-8817-4256
}

\author{
Stefanie Del Rocío Tutiven Sánchez \\ stefytutiven@gmail.com \\ ORCID: 0000-0001-8985-1669
}

\author{
Steven Miguel Calle Aveiga \\ steven96_08@hotmail.com \\ ORCID: 0000-0001-9366-0187
}

\section{Layla Yenebi De La Torre Ortega \\ layla_delatorre@yahoo.com ORCID: 0000-0002-4813-6957}

\author{
Isabel Odila Grijalva Grijalva \\ isabel.grijalva@cu.ucsg.edu.ec \\ ORCID: 0000-0003-4491-4149 \\ Universidad Católica de Santiago de Guayaquil, Ecuador \\ Recibido 21 de octubre 2020 | Arbitrado y aceptado 12 de noviembre 2020 | Publicado en 04 de enero 2021
}

\section{RESUMEN}

La Organización Mundial de la Salud (OMS), en el año 2018 reporta a la leucemia linfoblástica aguda (LLA) como principal causa de mortalidad en niños y adolescentes en el rango de edad de 0 a 19 años, cada año se diagnostican 300.000 casos nuevos. Objetivo: Determinar los efectos de los ejercicios de bajo impacto en la prevención de las complicaciones asociadas a la inactividad física en niños y niñas con Leucemia en el Hospital Oncológico SOLCA de la ciudad de Guayaquil. Material y métodos: El alcance de la investigación es descriptivo, observacional con diseño no experimental y de corte transversal. Los datos fueron obtenidos mediante el test de Cpax y la escala de fatiga, aplicada a 57 pacientes. Resultados: La evaluación por el test Cpax presentó que un 81\% tenían una buena condición física y un $19 \%$ presentó una condición regular; en relación a la saturación de oxígeno post evaluación un $98 \%$ presentaron valores superiores a $>90$ y un $2 \%$ valores $<90$; con respecto a la fatiga inicial se reportó un $49 \%$ con fatiga moderada, $42 \%$ fatiga extrema, $4 \%$ fatiga leve, $4 \%$ sin fatiga y $2 \%$ con la peor fatiga mientras que en la post evaluación un $51 \%$ presentó fatiga moderada, $35 \%$ fatiga leve, $11 \%$ fatiga extrema, $4 \%$ sin fatiga y $0 \%$ sin el nivel máximo de fatiga. El resultado de la prueba Chi-Cuadrado sobre la comparación Cpax pre y post evaluación, fue de $\mathrm{p}<0.05$ con un nivel de confianza del 99.9\% por lo que se presentó una significancia. Conclusión: Se evidenció el efecto positivo del plan de ejercicios de bajo impacto aplicado en pacientes con LLA.

Palabras clave: Leucemia linfoblástica aguda, fatiga, sedentarismo

\begin{abstract}
The World Health Organization (WHO), in 2018 reported acute lymphoblastic leukemia (ALL) as the main cause of mortality in children and adolescents in the age range from 0 to 19 years, each year 300,000 are diagnosed new cases. objective. To determine the effects of low-impact exercises in the prevention of complications associated with physical inactivity in boys and girls with Leukemia at the SOLCA Oncological Hospital in the city of Guayaquil. Material and methods. The scope of the research is descriptive, observational with a non-experimental and cross-sectional design. The data were obtained using the Cpax test and the fatigue scale, applied to 57 patients. Results. The evaluation by the Cpax test showed that $81 \%$ had a good physical condition and $19 \%$ had a regular condition; in relation to postevaluation oxygen saturation, $98 \%$ showed values higher than $>90$ and $2 \%$ values $<90$; Regarding the initial fatigue, $49 \%$ were reported with moderate fatigue, $42 \%$ extreme fatigue, $4 \%$ mild fatigue, $4 \%$ without fatigue and $2 \%$ with the worst fatigue, while in the post-evaluation $51 \%$ presented moderate fatigue, $35 \%$ mild fatigue, $11 \%$ extreme fatigue, $4 \%$ without fatigue and $0 \%$ without the maximum level of fatigue. The result of the Chi-Square test on the Cpax comparison before and after the evaluation was $\mathrm{p}<0.05$ with a confidence level of $99.9 \%$, therefore, a significance was presented. Conclusion. The positive effect of the low-impact exercise plan applied in patients with ALL was evidenced.
\end{abstract}

Key words: Acute lymphoblastic leukemia, fatigue, sedentarism
MA: Licenciada en Terapia física en el 2019 . Experiencia en protocolo de ejercicios de bajo impacto para pacientes oncológicos, traumatología. Actualmente labora en la Clínica Guayaquil en el área de UCI. Universidad Católica de Santiago de Guayaquil, Ecuador.

ST: Docente Titular Auxiliar 2 de la carrera de Fisioterapia. Coordinadora Académica de la carrera de Fisio. Universidad Católica Santiago de Guayaquil, Ecuador.

JCH: Licenciada en Terapia física de la Universidad Católica Santiago de Guayaquil, Ecuador. SC: Licenciado en Terapia Física. Experiencia en
protocolo de ejercicios de bajo impacto para pacientes oncológicos. Actualmente labora en el pentro Médico Club de Leones Urdesa, centro de atención Redima, Centro médico Medigrup. Especializado en Electro punción en fisioeducate Manta. Universidad Católica de Santiago de Guayaquil, Ecuador.

MB: Licenciada en Terapia Física y rehabilitación. Jefa del Departamento de terapia física y rehabilitación del Centro del Dolor. Aprobada para Therapeutic Pain Specialist la Asociación Americana de Terapia física y socia del Colegio de Fisioterapeutas del Guayas. Universidad Católica de Santiago de Guayaquil, Ecuador.

LT: Especialista en Fisioterapia en el Adulto Crítico. Licenciada en Terapia Física y rehabilitación. Magister en Educación Superior. Actualmente: Docente de la Carrera de Fisioterapia de la Universidad Católica de Santiago de Guayaquil. Fisioterapeuta Hospital General Guasmo Sur. Universidad Catolica de

SG: Doctora en Medicina y Cirugía, Universidad de Guayaquil. Magister en Salud Pública Universidad de Guayaquil. En la actualidad realizando PHD con la Universidad de Granada, España. Pertenece al grupo de investigación de Ciencias de la Salud de la UCSG. Docente Universitaria de la Universidad Católica Santiago de Guayaquil, Ecuador. 
MA: Licenciada en Terapia física en el 2019. Experiencia en protocolo de ejercicios de bajo impacto para pacientes oncológicos traumatologia. Actualmente labora en la Clínica Católica de Santiago de Guayaquil, Ecuador.

ST: Docente Titular Auxiliar 2 de la carrera de Fisioterapia. Coordinadora Académica de la carrera de Fisio. Universidad Católica Santiago de Guayaquil, Ecuador

JCH: Licenciada en Terapia física de la Universidad Católica Santiago de Guayaquil, Ecuador.

SC: Licenciado en Terapia Física. Experiencia en protocolo de ejercicios de bajo impacto para pacientes oncológicos. Actualmente labora en e Centro Médico Club de Leones Urdesa, centro de atención Redima, Centro médico Medigrup. Especializado en Electro punción en fartucar Manta. Univeridad Catica de Santiago de Guayaquil, Ecuado

MB: Licenciada en Terapia Física y rehabilitación. Jefa del Departamento de terapia física y rehabilitación del Centro del Dolor. Aprobada para Therapeutic Pain Specialis Certification-Spring. Miembro internacional de la Asociación Americana de Terapia física y socia del Colegio de Fisioterapeutas del Guayas. Universidad Católica de Santiago de Guayaquil, Ecuador

LT: Especialista en Fisioterapia en el Adulto Crítico Licenciada en Terapia Física y rehabilitación. Magister en Educación Superior. rehabilitación. Magister en Educación Superior Actualmente: Docente de la Carrera de Santiago de Guayaquil. Fisioterapeuta Hospital General Guasmo Sur. Universidad Católica de Santiago de Guayaquil, Ecuador.

SG: Doctora en Medicina y Cirugía, Universidad de Guayaquil. Magister en Salud Pública Universidad de Guayaquil. En la actualidad realina Perten con la Universida de Granán, Ciencias de a salud de investigación de Universitaria de la Universidad Dálica Santiago de Guayaquil, Ecuador.

\section{INTRODUCCIÓN}

$\mathrm{E}$ l cáncer infantil corresponde a un grupo de enfermedades relacionadas, las mismas que se presentan con un cambio celular el todo el cuerpo, estas células cancerosas empiezan una replicación sin detenerse hasta ubicarse en los tejidos cercanos. El cáncer infantil puede ocurrir de forma repentina, sin presentar síntomas y tiene una tasa de recuperación de $80 \%$ en países de ingresos altos y de $20 \%$ en países de bajos ingresos. El tipo de cáncer más frecuente en niños es la leucemia linfoblástica aguda (LLA), y otras presentaciones del cáncer infantil incluyen al sarcoma de tejido blando, linfoma y tumores cerebrales.(1).

Alarcón Ariel en su libro "Vencer el cáncer" expone que existen varios factores que pueden producir el cáncer como el estrés que es considerado como un resultado negativo de un conflicto entre el sistema inmunológico y la replicación celular inadecuada o desordenada.(2).

La LLA es la neoplasia de mayor presentación en la edad pediátrica, se estima que a nivel mundial se presenta de 20 a 35 casos por millón de habitantes al año; corresponde a entidades oncohematológicas que se presentan por invasión descontrolada de leucocitos inmaduros en medula ósea y sangre e infiltración en los tejidos. El cuadro clínico se compone de fiebre, anemia, hemorragias, dolor óseo y en articulaciones, fatiga, equimosis y petequias.(3).

La Agencia Internacional de Investigación del Cáncer (IARC) presentó los datos actualizados sobre incidencia, mortalidad y prevalencia de cáncer en el 
mundo, según su base de datos hasta el año 2012, existió 14.1 millones de nuevos casos y 7.6 millones de muertes por distintos tipos de cáncer. La situación del cáncer en América representa la segunda causa de muerte, en el año 2018 se pudo diagnosticar 3.8 millones de casos y 1.4 millones fallecieron por esta patología, en un 57\% ocurrieron en personas de 69 años o menos.(4).

Los pacientes que se encuentran en tratamiento: quimioterapia citotóxica, radioterapia o trasplante de médula ósea desarrollan un síndrome de fatiga muscular, que es una sensación subjetiva de debilidad, cansancio o falta de energía que afecta su calidad de vida y no le permite realizar las actividades diarias. La fatiga se vuelve en el síntoma más angustiante y que puede causar depresión al paciente oncológico.(5).

Se desconoce la patogenia o etiología de la fatiga relacionada con el cáncer, pero se han mencionado varios mecanismos como la desregulación de citoquinas pro y antiinflamatorias, funcionamiento del eje hipotálamo pituitaria-adrenal, acción del sistema monoaminas, alteración del ritmo circadiano y el metabolismo energético muscular. En los niños con LLA y otros tipos de cáncer, por el síndrome de fatiga tienen dificultad para jugar o concentrarse, generando pensamientos negativos como ira, enojo o ansiedad; por lo que se debe emplear alternativas terapéuticas. (6).

Se ha considerado la práctica de ejercicios para mejorar la condición física y mental de los pacientes oncológicos.(7).

En el Hospital Oncológico donde se realizará el estudio, se aprecian complicaciones debido a la actividad física posterior a la quimioterapia por lo que se considera necesario evaluar los resultados favorables de los ejercicios de bajo impacto en pacientes con Leucemia Linfoblástica Aguda.

La finalidad del presente artículo será determinar los efectos de los ejercicios de bajo impacto para prevenir complicaciones asociadas a la inactividad física producida posterior al tratamiento terapéutico en niños y niñas con LLA en un Hospital Oncológico de Guayaquil-Ecuador en el periodo 2020. El grupo de estudio está conformado por pacientes oncológicos de pediatría, se realizó una evaluación luego de su tratamiento de quimioterapia con la aplicación de la prueba de funcionalidad física (Cpax) y el grado de fatiga, posteriormente se ejecutan los ejercicios de bajo impacto y se verifican sus efectos de acuerdo a cada parámetro evaluado.(8).

\section{MATERIALES Y MÉTODOS}

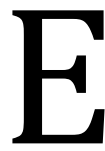
l presente trabajo de investigación es de alcance descriptivo de enfoque cuantitativo con un diseño no experimental de corte transversal, el mismo que se apoyó en una base de datos de tipo numérico y aplicación de instrumentos como test o escalas sobre condición física para responder a los objetivos e hipótesis planteadas. Los instrumentos utilizados en esta investigación corresponden a la Escala de fatiga y test Cpax. El cuestionario Cpax representa una puntuación para determinar la funcionalidad física, está compuesto por 10 parámetros a evaluarse: la función respiratoria, tos, movilidad en cama, posición decúbito supino a sentado en el borde de la cama, equilibrio de pie, sentado dinámico, sentado para pararse, traspaso de cama a silla, los pasos y la fuerza de agarre.(9). 
La población del estudio son los pacientes pediátricos con LLA que fueron sometidos a quimioterapia, que se encuentran en el grupo etáreo de 0-15 años de edad en el Hospital Oncológico SOLCA de la ciudad de Guayaquil; de acuerdo con los criterios de inclusión, se estableció la muestra de 57 pacientes oncológicos a los cuales se les aplico los instrumentos de evaluación.(10).

El trabajo fue realizado en el Hospital Oncológico SOLCA de la ciudad de Guayaquil por un equipo de profesionales de la Carrera de Terapia Física de la Universidad Católica Santiago de Guayaquil en el año 2019, teniendo la autorización del mismo para desarrollar el estudio en el establecimiento. Se evaluó de forma visual a cada uno de los pacientes pediátricos para establecer la muestra (57 pacientes), así como la condición de riesgos o complicaciones.

Los ejercicios empleados fueron la técnica Mindfulness que corresponde a ejercicios de relajación o respiración para conseguir el bienestar en los pacientes luego de la quimioterapia, este tratamiento causa fatiga o dolor; sumado a esta técnica, se incluyen ejercicios de bajo impacto o combinados con patrones respiratorios controlados. (11).

El análisis e interpretación de datos se realizó por estadística descriptiva; específicamente la distribución de edad y sexo expresada por porcentaje y los datos numéricos de los parámetros evaluados del Test Cpax.

\section{RESULTADOS}

$\mathrm{E}$ n los 57 pacientes del estudio se establece la clasificación por edad y sexo, se observa una mayor frecuencia del sexo masculino y en la edad comprendida de 6 a 10 años. En lo que respecta al sexo femenino; se observa el $57 \%(n=13)$ en el grupo etáreo de 6 a 10, el $35 \%(n=8)$ al rango de edad de 11 a 15 y el $9 \%(\mathrm{n}=2)$ al rango de 0 a 5 años. En el sexo masculino, la distribución es el 53\% ( $\mathrm{n}=18)$ en el grupo etáreo de 6 a 10 años, 26\% $(n=9)$ en el rango de 0 a 5 y el $21 \%(n=7)$ en el rango de 11 a 15.

Con la aplicación del Test Cpax se evidencia la funcionalidad física de los pacientes oncológicos pediátricos del Hospital SOLCA. En el diagrama de Cajón y Bigotes, se puede establecer los cambios pre y post evaluación con los resultados obtenidos por el test. En la pre evaluación se obtuvo un valor promedio de 43.089 puntos, siendo el valor máximo 50 y el mínimo 19, en lo que respecta a la post evaluación se obtiene un promedio de 46.57 puntos con el mismo valor máximo de 50 y el mínimo es de 30 . Se puede evidenciar el impacto favorable de los ejercicios en la condición física, descartando puntuaciones menores a 30. (Gráfico 1). 


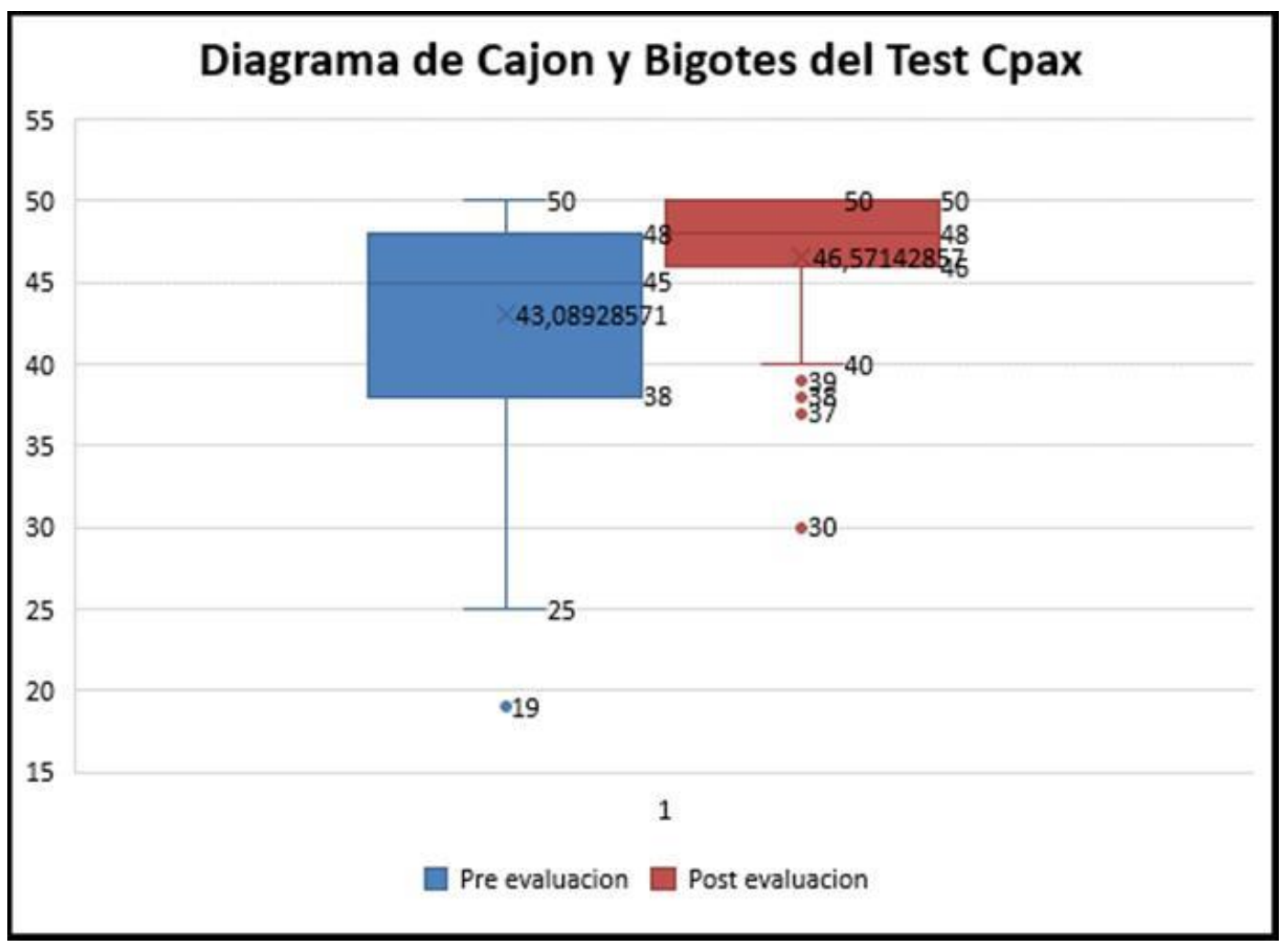

Gráfico 1. Resultado del Test Cpax

En el análisis de los parámetros de Cpax se evaluó la función respiratoria en la muestra del estudio; en la pre evaluación se obtuvo el prmedio de 9.5 puntos con un valor máximo de 47 y mínimo de 0 , mientras que en la post evaluación el promedio fue 9.5 puntos con el valor máximo de 49 y el mínimo de 0 . Se puede establecer que la función respiratoria no presento modificaciones. (Gráfico 2). 


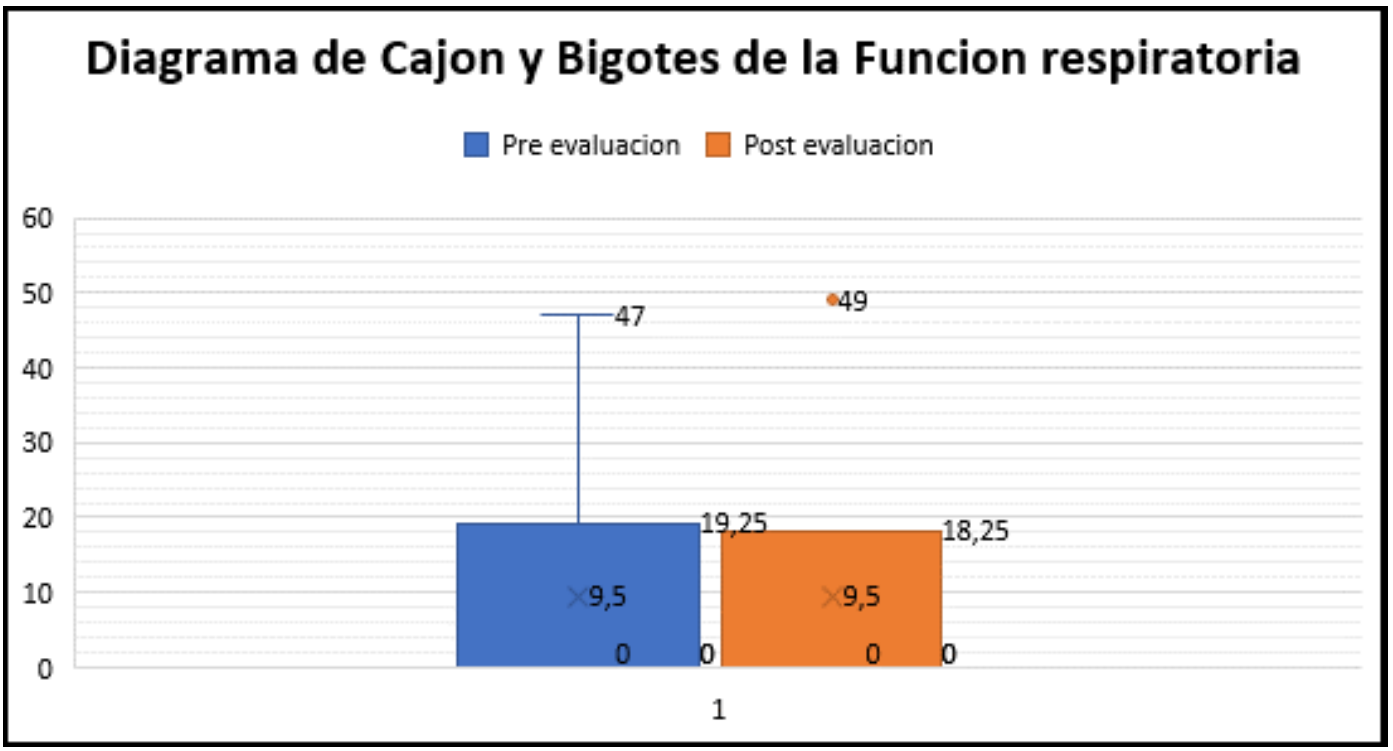

Gráfico 2. Resultados de función respiratoria

En la realización del Test Cpax se evaluó la tos en los pacientes oncológicos pediátricos; en la pre evaluación se obtuvo el promedio de 9.5 puntos con un valor máximo de 32 y mínimo de 0 , mientras que en la post evaluación el promedio fue 9.5 puntos con el valor máximo de 37 y el mínimo de 0 . El parámetro Tos se mantuvo con relación a la pre evaluación. (Gráfico 3).

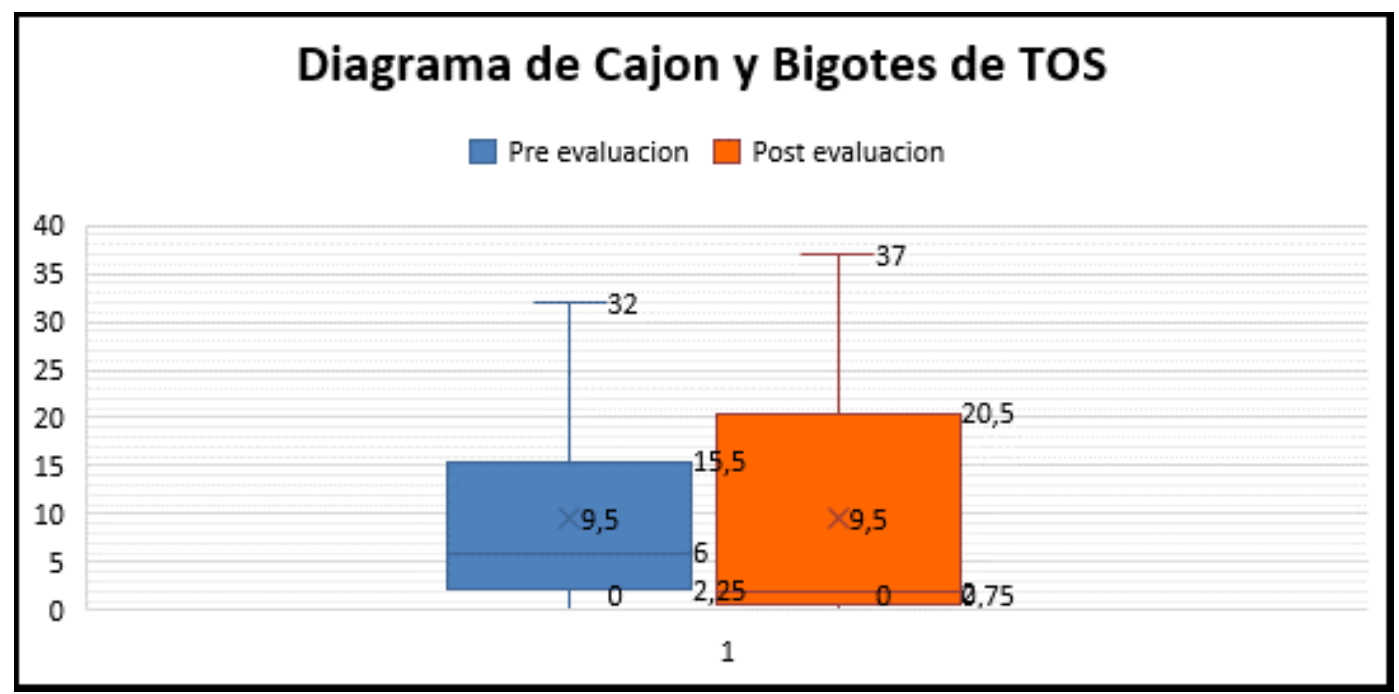

Gráfico 3. Resultados del reflejo de tos

En el análisis de los parámetros Cpax se evaluó la prensión manual en los pacientes del estudio; en la pre evaluación se obtuvo el promedio de 9.5 puntos con un valor máximo de 18 y mínimo de 0 , mientras que en la post evaluación el promedio fue 9.5 puntos con el valor máximo de 21 y el mínimo de 0 . La prensión manual no se modificó con relación a la pre evaluación. (Gráfico 4). 


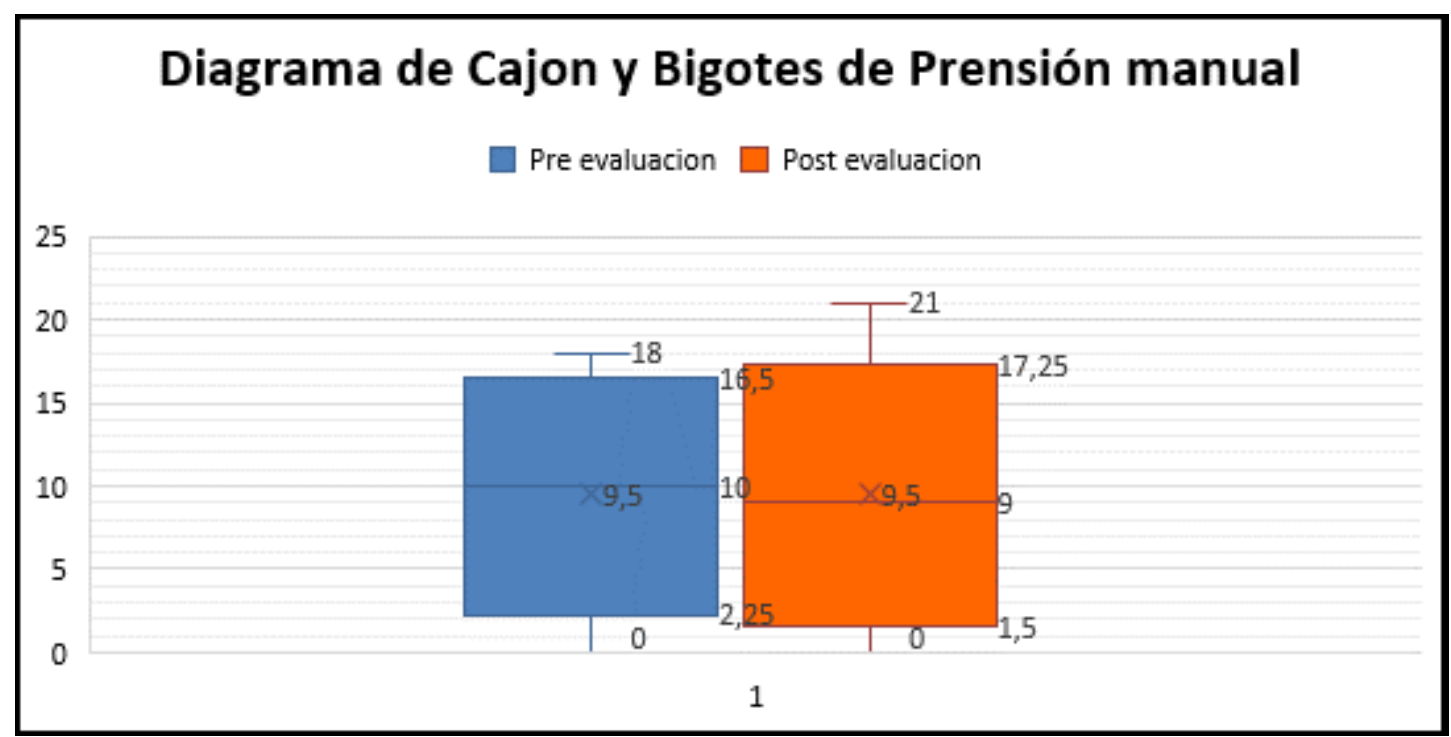

Gráfico 4. Resultados de prensión manual

Se realizó una preevaluación de fatiga en donde se obtuvo que el $49 \%$ de la muestra con fatiga moderada, $42 \%$ fatiga extrema, $4 \%$ en el puntaje de fatiga leve, $4 \%$ no tenía fatiga y el $2 \%$ con la peor fatiga. En la post evaluación se evidencian cambios notorios, el $51 \%$ presentó fatiga moderada, el $35 \%$ fatiga leve, un $11 \%$ fatiga extrema, el $4 \%$ no tuvo fatiga y no se observó ningún integrante de la muestra en el nivel de peor fatiga. (Gráfico 5).

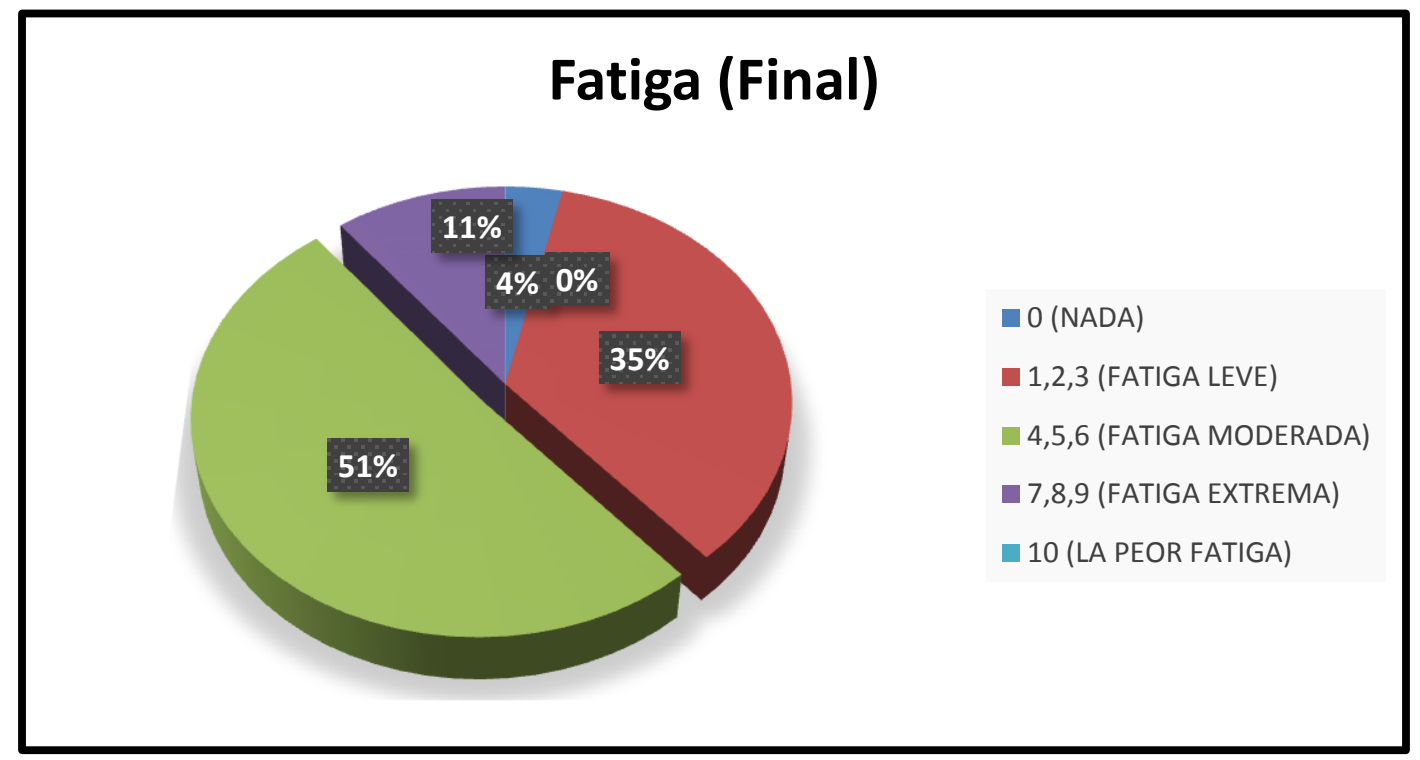

Gráfico 5: Resultado post evaluación de fatiga por puntuación. 
Se realizó el análisis de la saturación de oxígeno en los pacientes del área de pediatría del Hospital SOLCA; en la preevaluación se obtuvo como valor promedio 93.92 puntos, siendo el máximo 99 y 78 el mínimo, con respecto a la post evaluación, el valor promedio fue de $96.96 \%$ con un máximo valor de $100 \%$ y mínimo de $88 \%$. Se puede observar un mejor resultado en la saturación de oxígeno con respecto en los valores de la pre evaluación. (Gráfico 6).

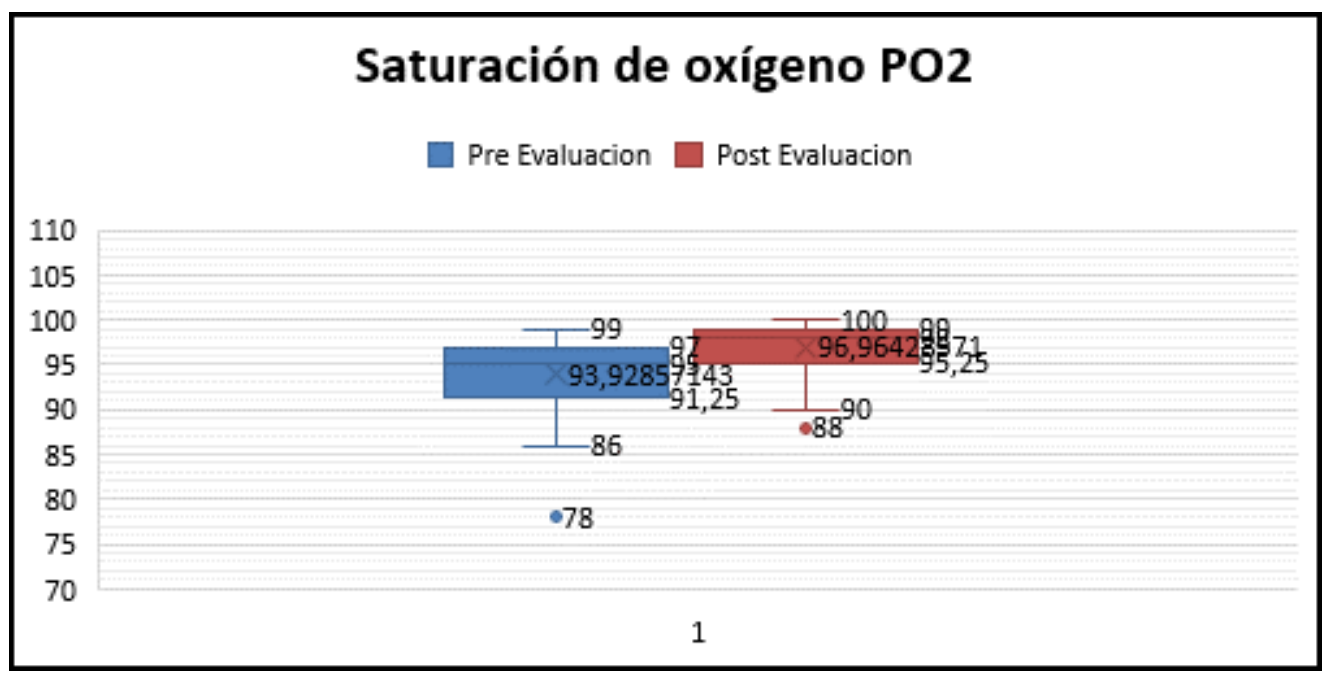

Gráfico 6. Resultado de Saturación de Oxígeno

\section{Discusión}

La Leucemia Linfoblástica Aguda ha presentado en los últimos años una cifra de supervivencia del $90 \%$ en países desarrollados por los avances terapéuticos y eficacia asistencial. Existen varios efectos por el diagnóstico de la enfermedad o tratamientos estresantes como la quimioterapia, entre las repercusiones se encuentra la osteopenia, alteraciones motoras y atrofia muscular que se asocia a la inactividad física.

Se ha realizado una búsqueda exhaustiva de artículos y estudios nacionales o internacionales realizados por la comunidad científica sobre la influencia de ejercicios físicos para prevenir complicaciones en pacientes con leucemia, no se encontraron trabajos sobre el tema.

Sobre la distribución de la edad y sexo de los pacientes oncológicos, se evidenció el predominio del sexo masculino y el grupo etario de 6-10 años; lo que concuerda con el estudio realizado por Agurto María (2019) denominado " Efectividad de los ejercicios de bajo impacto en la prevención de las complicaciones asociadas a la inactividad física en niños y niñas con leucemia que asisten al Hospital SOLCA de la ciudad de Guayaquil" en el que reportó $53 \%$ de pacientes masculinos en la edad de 6 a 10 años. Se ha indicado que el sexo masculino tiene mayor predisposición a desarrollar cáncer por su constitución cromosómica o genética.(8)

Con el diagrama Cajón y Bigotes se establece la efectividad de los ejercicios empleados en los pacientes con leucemia; en la preevaluación se obtuvo un promedio de 43 puntos con un valor máximo de $50 \mathrm{y}$ mínimo de 19, mientras que en la evaluación posterior el promedio fue de 46 
puntos con el máximo de 50 y mínimo 30; es decir que descartamos condiciones físicas bajas. Uclés Vanessa (2017) en su artículo "Prescripción del ejercicio en el paciente con cáncer" sugiere que la calidad de vida se mejora aumentando la capacidad física o funcional, mejora el síndrome de fatiga y la depresión en pacientes sometidos a quimioterapia; la rutina de ejercicios moderado puede ser de 90 a 120 minutos por semana. (12).

En la post evaluación se registraron cambios en la función respiratoria obteniendo el $86 \%$ en un nivel 5 , la tos en el nivel 5 en un 88\%, con respecto a la prensión manual el 37\% alcanzó el nivel 5, $28 \%$ el nivel $4,28 \%$ el nivel 3, un $4 \%$ el nivel 2; además se reporta mejoría de la saturación de oxígeno con un promedio de 96.9\%. Battaglini Claudio (13) en su artículo de revisión "Efectos del ejercicio físico sobre el cáncer" expone sus beneficios: mejora la función pulmonar, cardiovascular y muscular; incrementa el volumen de eyección cardiaca, tono muscular, ritmo metabólico, la coordinación y el balance. (13).

En la evaluación de fatiga en nuestro estudio, al realizar el test Cpax inicial se reportó $49 \%$ de pacientes con fatiga moderada, $42 \%$ con fatiga extrema, un $4 \%$ de fatiga leve y $2 \%$ con la peor fatiga; en la post evaluación se reporta disminución de casos de fatiga extrema con un $11 \%$, la fatiga leve presente en un 35\% y no hubo pacientes con la peor fatiga. Vargas Celina (2015) en su artículo "Síndrome de fatiga relacionado con cáncer" expone que el ejercicio físico es una intervención no farmacológica para el síndrome de fatiga en pacientes sometidos a quimioterapia, probablemente porque se disminuyen las citoquinas proinflamatorias y aumenta la actividad linfocítica NK por la actividad física. (14).

\section{CONCLUSIONES}

$\mathrm{S}$ e realizó la distribución porcentual de los 57 pacientes pediátricos con Leucemia atendidos en el Hospital Solca de la ciudad de Guayaquil, según el sexo y la edad, se observa una mayor frecuencia del sexo masculino con un $53 \%$ ( $n=18$ niños) en el rango de edad de 6 a 10 años.

La aplicación del test Cpax (Chelsea Critical Care Physical Assement Tools) permitió la recolección de datos con respecto a la condición física antes $\mathrm{y}$ después de aplicar los ejercicios de bajo impacto. Entre los resultados de mayor relevancia se encuentran, lo que respecta a fatiga, modificó en la post evaluación eliminando el $2 \%$ de pacientes que cursaban el nivel 10 o peor fatiga, disminuyó los casos de fatiga extrema de $42 \%$ a $11 \%$, el $86 \%$ de los pacientes presentaron fatiga leve o moderada.

Se ejecutó la estrategia fisioterapéutica en base a ejercicios en niños y niñas con leucemia que se encuentran en tratamiento de quimioterapia; el plan estuvo compuesto por ejercicios de estiramiento, respiración, flexibilidad, bajo impacto y combinado con patrones respiratorios controlados. El programa de ejercicios se realizó tres veces con juegos de relajación, en los niños siempre se mantuvo el control de la frecuencia cardiaca, presión arterial y saturación de oxígeno.

En la post evaluación se pudo establecer la mejoría de los valores de saturación de oxígeno, obteniendo cifras de $88 \%$ a $100 \%$ lo que mejora el sueño de los pacientes y evita problemas respiratorios como disneas. 


\section{REFERENCIAS BIBLIOGRÁFICAS}

1. López A, Frías V, López M, García I, Alonso P, Atienza A. Programa de formación continuada en Pediatría Extrahospitalaria. SEPEAP. agosto de 2016;XX(6):97

2. Alarcón Prada A. Vencer el Cáncer. Bogotá: Colombia Intermedio; 2012

3. Lassaletta A. Leucemias. Leucemia linfoblástica aguda [Internet]. SEPEAP. 2012 [citado 6 de enero de 2021]. Disponible en: https://www.pediatriaintegral.es/numeros -anteriores/publicacion-2012-

07/leucemias-leucemia-linfoblasticaaguda/

4. Mitchell C. OPS/OMS | La OMS describe los pasos para salvar 7 millones de vidas amenazadas por el cáncer [Internet]. Pan American Health Organization / World Health Organization. 2020 [citado 6 de enero de 2021]. Disponible en: https://www.paho.org/hq/index.php?optio $\mathrm{n}=$ com_content\&view=article\&id=15708: $\mathrm{w}$ ho-outlines-steps-to-save-7-million-livesfrom-cancer\&Itemid=1926\&lang=es

5. Vargas C. Síndrome de fatiga relacionado con Cáncer. Rev Clinica Escuela Medicina UCR. 2015;5:7

6. Salas M, Gabaldón O. Evaluación y control de síntomas en oncología pediátrica: Una necesidad que cubrir y un mundo por descubrir. PsicoOncología. 2016;1(2-3):231-50

7. Meneses J, González E, Correa J, Valle J, Ramírez R. Efectividad del ejercicio físico en la fatiga de pacientes con cáncer durante el tratamiento activo: revisión sistemática y metaanálisis. Cad Saúde Pública. abril de 2015;31(4):667-81.

8. Agurto $M$, Calle S. Efectividad de los ejercicios de bajo impacto en la prevención de complicaciones asociadas a la inactividad física en niños y niñas con leucemia que asisten al Hospital Solca en
Guayaquil [Internet] [Tesis de grado]. [Guayaquil-Ecuador]: Universidad Católica Santiago de Guayaquil; 2019. Disponible en: http://repositorio.ucsg.edu.ec/bitstream/3 317/12626/1/T-UCSG-PRE-MED-TERA181.pdf

9. Corner E, Wood H, Englebretsen C, Thomas A, Grant R, Nikoletou D, et.al. The Chelsea Critical Care Physical Assessment Tool (CPAx): validation of an innovative new tool to measure physical morbidity in the general adult critical care population; an observational proof-of-concept pilot study. Physiotherapy. marzo de 2013;99(1):33-41

10. Font A, Rodríguez E, Buscemi V. Fatiga, expectativas y calidad de vida en cáncer. PsicoOncologia. 2016;1(2-3):45-56

11. Vásquez E. Mindfulness: Conceptos generales, psicoterapia y aplicaciones clínicas. Rev Neuropsiquiatr. 4 de abril de 2016;79(1):42

12. Uclés V, Espinoza R. Prescripción del ejercicio en el paciente con cáncer. Rev Clinica Escuela Medicina UCR. 2017;7(II):8

13. Battaglini C. Efectos del ejercicio físico sobre el cáncer. Rev Digital Buenos Aires [Internet]. 2003;(61). Disponible en: https://www.efdeportes.com/efd61/cance r.htm

14. Vargas C. Síndrome de fatiga relacionado con el Cáncer. Rev Clinica Escuela Medicina UCR. 2015;5(IV):7

Conflicto de intereses. Los autores declaran que no existe conflicto de intereses para la publicación del presente artículo.

Financiamiento. Autofinanciamiento

Agradecimiento. No declaran 